\title{
3D ultrasound and HD live of an ovarian mucinous cystadenoma
}

\section{Mihaela Grigore*, Flavius Iordache, Dumitru Gafitanu}

Department of Obstetrics \& Gynaecology, University of Medicine and Pharmacy, Grigore T. Popa, Iasi, Romania

Received: 10 December 2015

Accepted: 08 January 2016

*Correspondence:

Dr. Mihaela Grigore,

E-mail: mihaela.grigore@edr.ro

Copyright: $\odot$ the author(s), publisher and licensee Medip Academy. This is an open-access article distributed under the terms of the Creative Commons Attribution Non-Commercial License, which permits unrestricted non-commercial use, distribution, and reproduction in any medium, provided the original work is properly cited.

\begin{abstract}
Ovarian mucinous cystadenoma is a benign tumor that arises from the surface epithelium of the ovary. We present a case of a bilateral mucinous adenoma in a 19 years old patient. The 2D ultrasound was used in combination with 3D and HD live images. The preoperative diagnosis according to ultrasound images was mucinous adenoma and the pathological report after surgery confirmed it. HD live is a novative ultrasound software which can improve the diagnosis.
\end{abstract}

Keywords: Ovarian mucinous cystadenoma, 3D ultrasound, HD live

\section{INTRODUCTION}

Ovarian mucinous cystadenoma is a benign tumor that arises from the surface epithelium of the ovary. Benign mucinous cystadenoma represents $15 \%$ of all benign ovarian neoplasms and up to $80 \%$ of all ovarian mucinous tumors. It is common between the third and the fifth decades and is rare at the extremities of ages, before puberty and after menopause. ${ }^{1}$ On gross appearance, mucinous tumors are characterized by cysts of variable sizes without surface invasion.

\section{CASE REPORT}

The paper presents a case of a 19 years old girl who was admitted in the hospital for abdominal pain and abdominal enlargement. The clinical examination revealed a large abdominal mass developed through the whole abdomen. The pregnancy test was negative. Abdominal sonographic evaluation was performed using a Voluson E8 Expert (GE Healthcare) with volume convex abdominal and endovaginal probes (RAB6-D,
RIC5-9D). The examination demonstrated a multiloculated pelvic and abdominal mass which ocuppied the whole abdomen (Figure 1). As part of the examination, 3D volumes were obtained using abdominal and transvaginal probes and stored (Figure 2). The region of interest was selected in order to include the targeted structure and volume rendering was performed in subsequent offline analysis. A transvaginal sonographic assessment of the cyst using the same ultrasound machine revealed the origin of the tumor being on the right ovary and also detected a $4 \mathrm{~cm}$ cyst on the left ovary (Figure 3). The ultrasound machine equipped with a new surface render mode (HD live) allowed pictures of the tumour with this soft (Figure 4 and 5). The tumoral markers CA125, CA 19-9 were in normal range. According to the sonographic appearance of the pelvic mass, a preoperative diagnosis of ovarian mucinous cystadenoma was suggested. Right ovariectomy and left cystectomy were performed. The postoperative recovery was uneventful. The larger tumor measured $25 \times 10 \times 15 \mathrm{~cm}$ and weighted $5.5 \mathrm{~kg}$. The pathological report described mucinous cystadenoma of intestinal type. 


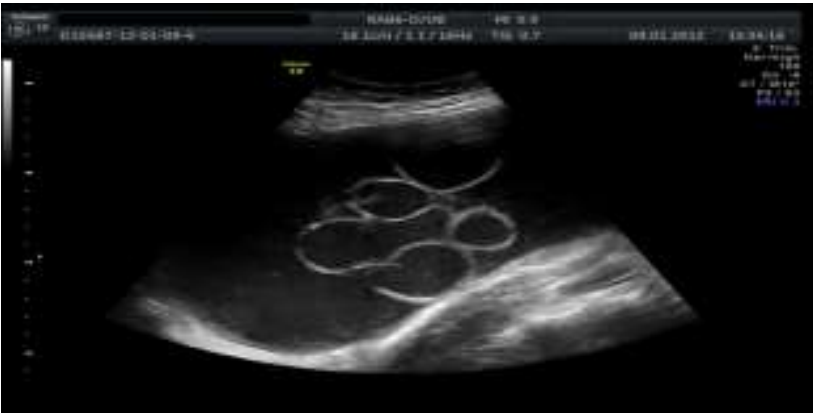

Figure 1: A multilocular cyst containing fluid of different echogenicities, with regular wall and septa, and no vegetations. The absence of solid components suggests a benign tumor.

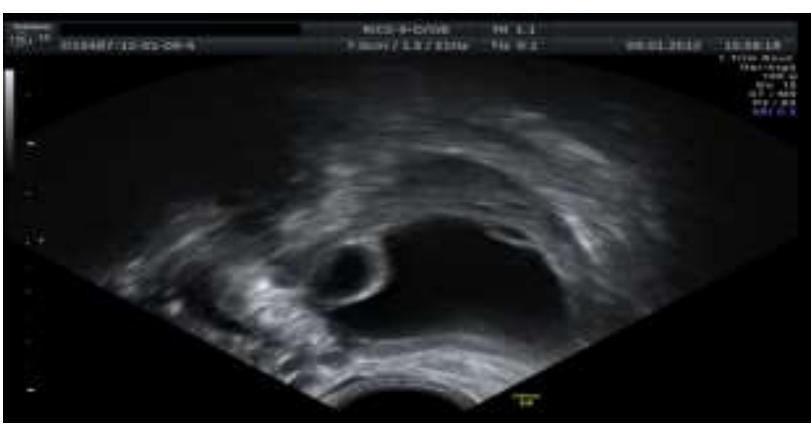

Figure 2: Endovaginal view of the left ovary. A cystic mass with a fine septation is visible.

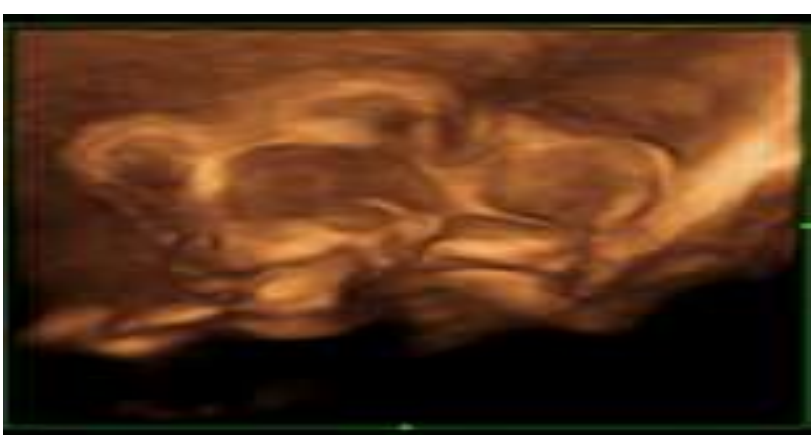

Figure 3: 3D image of the cystic mass with a network of fine septations. The arrangements of the septations are clearly displaced.

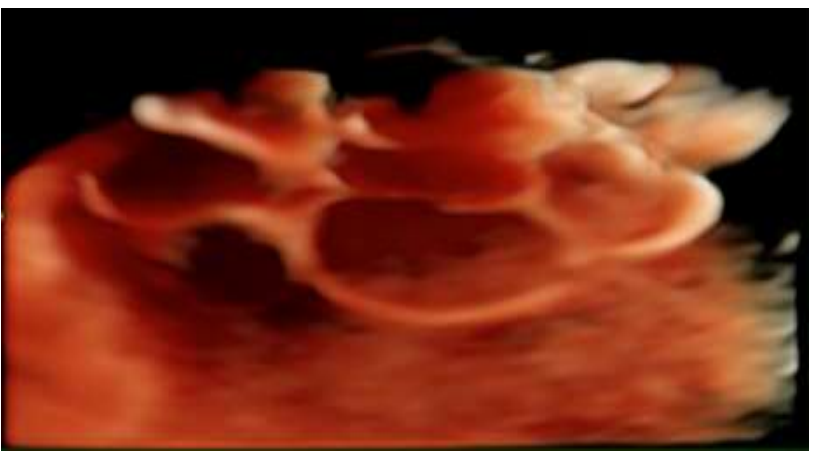

Figure 4: HD live of the cyst. The septations are better view with this soft.

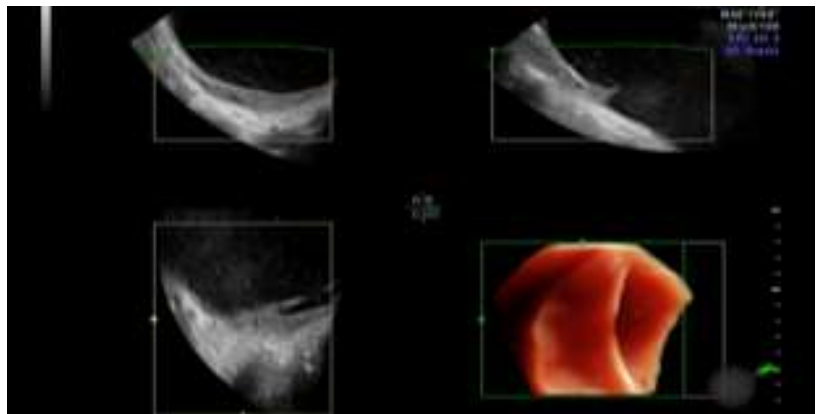

Figure 5: HD live image of the interior wall of the cyst. A fold of the wall is clearly visible.

\section{DISCUSSION}

Benign mucinous cystadenomas account for about $15 \%$ of all ovarian neoplasms. An ovarian mucinous cystadenoma does not usually cause symptoms until it becomes very large. Small tumors are often found incidentally, whereas large tumors present as an obvious pelvic or abdominal mass.

The most frequent complications of benign ovarian cysts are torsion, haemorrhage and rupture. As it contains mucinous fluid, the rupture of a mucinous cystadenoma leads to mucinous deposits on the peritoneum. The rupture and the spillage of the mucinous content could appear during surgery. The spillage of the cyst could lead to pseudomixoma peritonei and also carry the risk of recurrence in the benign mucinous cyst as well. ${ }^{2}$ The preoperative diagnostic of an ovarian mucinous cystadenoma is of outmost importance in order to prevent this complication. Imaging methods, particularly ultrasound imaging are almost always used to determine the nature of an adnexal mass. ${ }^{3}$ The sonographic appearance of mucinous cystadenomas typically includes numerous septations and frequently contains fine, gravity-dependent echoes produced by thick contents. The ultrasound examiner often misdiagnose mucinous cyst as serous cyst because the ultrasound morphology of serous and mucinous cystadenomas may overlap extensively. ${ }^{4}$ Also the ultrasound morphology of serous and mucinous cystadenomas overlaps to some extent that of the fibroadenomas. ${ }^{4}$ This difficulties demonstrate that using the pattern recognition represented by gray-scale evaluation and Doppler findings is not enough for a correct diagnosis. Three-dimensional ultrasound is a useful tool for ovarian pathology. The 3D inversion mode, which displays the cystic portions within the entire volume as echogenic areas could also be used. ${ }^{5}$

In the case presented, the tumor showed numerous septations localized in the inferior part of the large tumor. The 3D image of the rightt ovary was useful in observing the inner surface and content of the cyst. HD live software has been used in order to better view the content of the cyst. The HD live technology uses an adjustable light source, giving the operator the possibility to create lighting and shadowing effects and thereby increase 
depth perception. In both images it is clear to observe the possibility to adjust lighting. HD live was developed especially for obstetric usage because it gives a natural and realistic appearance of the fetus. In the present case this soft proved to be useful for examining the septations inside of the tumor. 3D or HD live views could clearly establish the presence of the loculation inside of the cyst. They are also useful tools for counting the loculi inside of the cyst. This counting is recommended because it was shown that the number of the loculi and the maximum diameter of the tumor might be alternate indicators for differentiating malignant from benign mucinous cystic neoplasms. $^{6}$

Two particularities must be underlined in the present case. First one is represented by the age of the patient, this type of tumor being very rare in the adolescence and secondly the bilateral character of the cysts. Because of the small size of one of these two cysts, the left ovary was preserved but assessing regular ultrasound examinations are planned in the first two years postoperative. $^{4,7-9}$

The 3D ultrasound and HD live used in conjunction to pattern recognition (gray-scale evaluation and Doppler findings) proved to be useful in examination of the cyst content. In complex adnexal mass, where it is difficult to discriminate between the types of tumor, 3D with additional softs could add interesting and useful information regarding the content of the tumor.

Funding: No funding sources

Conflict of interest: None declared

Ethical approval: Not required

\section{REFERENCES}

1. Ioffe OB, Simsir A, Silverberg SG. Pathology in Berek JS, Hacker NF (eds): Practical Gynaecologic
Oncology, editor. Lippincott Williams \& Wilkins Company; 2000: 213-4.

2. Ben-Ami I, Smorgick N, Tovbin J, Fuchs N, Halperin R, Pansky M. Does intraoperative spillage of benign ovarian mucinous cystadenoma increase its reccurence rate? Am J Obstet Gynecol. 2010;202:142.

3. Valentin L. Imaging in gynecology. Best Pract Res Clin Obstet Gynecol. 2006;20:881-906.

4. Sokalska A1, Timmerman D, Testa AC, Van Holsbeke C, Lissoni AA, Leone FP, et al. Diagnostic accuracy of transvaginal ultrasound examination for assigning a specific diagnosis to adnexal masses. Ultrasound Obstet Gynecol. 2009;34:462-70.

5. Grigore M, Mare A. Application of 3D ultrasound in female infertility. Rev Med Chir Nat Iasi. 2009;113:1113-9.

6. Okamoto Y, Tanaka YO, Tsunoda H, Yoshikawa H, Minami M. Malignant or borderline mucinous cystic neoplasms have a larger number of loculi than mucinous cystadenoma: a retrospective study with MR. J Magn Reson Imaging. 2007;26:94-9.

7. Ozgun MT, Turkyilmaz C. A giant ovarian mucinous cystadenoma in an adolescent: a case report. Arch Med Sci. 2009;5:281-3.

8. Alobaid AS. Mucinous cystadenoma of the ovary in a 12-year-old girl. Saudi Med J. 2008;29:126-8.

9. Kamel RM. A massive ovarian mucinous cystadenoma: a case report. Reproductive Biology and Endocrinology. 2010;8:24-5.

Cite this article as: Grigore M, Iordache F, Gafitanu D. 3D ultrasound and HD live of an ovarian mucinous cystadenoma. Int J Reprod Contracept Obstet Gynecol 2016;5:569-71. 